\title{
MIRNAS FROM SHRIMP PENAEUS MONODON AND ITS POSSIBLE ROLE IN INNATE IMMUNITY
}

\author{
Napol Kaewkascholkul', Kulwadee Somboonviwat ${ }^{3}$, Ikuo Hirono ${ }^{2}$, Anchalee \\ Tassanakajon $^{1}$, Kunlaya Somboonwiwat*
}

\author{
${ }^{I}$ Center of Excellence for Molecular Biology and Genomics of Shrimp, Department of \\ Biochemistry, Faculty of Science, Chulalongkorn University, Bangkok 10330, Thailand \\ ${ }^{2}$ Software Engineering Program, International College, King Mongkut's Institute of Technology \\ Ladkrabang, Chalongkrung Road, Ladkrabang, Bangkok 10520, Thailand \\ ${ }^{3}$ Graduate School of Marine Science and Technology, Tokyo University of Marine Science and \\ Technology, Tokyo 108-8477, Japan \\ ${ }^{4}$ Department of Aquatic Bioscience, Graduate School of Agricultural and Life Sciences, The \\ University of Tokyo, Tokyo 113-8657, Japan
}

\begin{abstract}
MicroRNAs (miRNAs) are short noncoding RNAs of the RNA interference pathways that regulate gene expression through partial complementary base pairing to target mRNAs. Here, miRNAs that are expressed in the white spot syndrome virus (WSSV)-infected Penaeus monodon hemocyte, were identified by next generation sequencing. Forty-six miRNA homologs were identified. Of those, the expression of 16 miRNAs in response to WSSV infection was analyzed by stem-loop real-time PCR. Eleven out of sixteen miRNAs were differently expressed upon WSSV infection. Two miRNAs, pmo-miR-315 and pmo-miR-750, were highly responsive upon WSSV infection. From the miRNA target prediction, miRNAs were targeted at 5'UTR, ORF, and 3'UTR of several immune-related genes involved in apoptosis, antimicrobial peptides, prophenoloxidase system, proteinase and proteinase inhibitor. To characterize the miRNA function, the highly conserved miRNA homolog, pmo-bantam, was further analyzed. A highly conserved miRNA, pmo-bantam was predicted to target the 3'UTR of an immune gene, Kunitztype serine protease inhibitor (KuSPI). Binding of pmo-bantam to the target sequence of KuSPI gene was confirmed by luciferase reporter assay. Also, correlation of pmo-bantam and KuSPI expression was revealed in lymphoid organ of WSSV-infected shrimp suggesting that the regulatory role of pmo-bantam in shrimp lymphoid organ. These results implied that miRNAs might play roles as immune gene regulators in shrimp antiviral response.
\end{abstract}

\section{KEYWORDS}

Penaeus monodon; microRNA; WSSV; bantam; antiviral response

*Corresponding author. Tel.: +66 22185438; Fax: +66 22185418.

E-mail address: kunlaya.s@chula.ac.th 